\title{
Las actitudes del profesorado de educación primaria hacia la educación inclusiva en Ecuador
}

\section{Resumen}

\author{
Raúl Tárraga-Mínguez ${ }^{1}$ \\ http://orcid.org/0000-0002-4458-5763 \\ Ximena Vélez-Calvo² \\ https://orcid.org/0000-0002-4451-9547 \\ Gemma Pastor-Cerezuela ${ }^{3}$ \\ https://orcid.org/0000-0002-4992-4701 \\ M. Inmaculada Fernández-Andrés ${ }^{\dagger 3}$ \\ https://orcid.org/0000-0003-2808-0949
}

Las actitudes de los docentes hacia la inclusión son un factor relevante para permitir que las políticas educativas inclusivas puedan materializarse verdaderamente en prácticas inclusivas. Estas actitudes se ven influenciadas por diferentes variables, entre las que se encuentran la formación y experiencia en el ámbito de la inclusión y las condiciones y recursos disponibles para llevar a cabo prácticas educativas. El objetivo del presente trabajo es analizar las actitudes hacia la inclusión educativa en una muestra de 66 docentes de 15 centros educativos de la ciudad de Cuenca (Ecuador), mediante una escala tipo Likert de 23 items. Los resultados muestran que los docentes presentan actitudes ambiguas y poco decididas hacia la inclusión educativa. Los docentes de mayor edad y sin experiencia docente con estudiantes con necesidades educativas especiales (NEE) presentaron peores actitudes que los docentes de menor edad y con experiencia docente con estudiantes con NEE. Además, los docentes de los centros educativos privados presentaron mejores actitudes hacia la inclusión que los de los centros públicos. Estos resultados sugieren que es necesario realizar un mayor esfuerzo en la formación del profesorado en el ámbito de la inclusión, y que se debería dotar a los centros públicos de recursos más adecuados para facilitar las posibilidades de llevar a cabo prácticas educativas verdaderamente inclusivas.

\section{Palabras clave}

Actitudes - Educación inclusiva - Experiencia docente - Formación del profesorado.

1- Universidad de Valencia,Valencia, España. Contacto: raul.tarraga@uv.es.
2- Universidad del Azuay, Cuenca, Ecuador. Contacto: xvelez@uazuay.edu.ec.
3- Universidad de Valencia,Valencia, España. Contacto: Gemma.Pastor@uv.es.

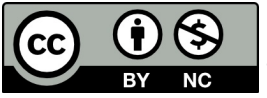




\section{Attitudes of elementary school teachers towards inclusive education in Ecuador}

Teachers' attitudes towards inclusion are a relevant factor in allowing inclusive educational policies to truly materialize in inclusive practices. These attitudes are influenced by different variables, such as training and experience in inclusion and the conditions and resources available to carry out educational practices. The objective of this study is to analyze the attitudes towards educational inclusion in a sample of 66 teachers from 15 educational centers in the city of Cuenca (Ecuador), using a 23-item Likert-type scale. The results show that teachers have ambiguous and undecided attitudes towards educational inclusion. Older teachers who had no experience in teaching special educational needs' (SEN) students, had worse attitudes than younger teachers who had already taught SEN students. Furthermore, teachers in private schools showed better attitudes towards inclusion than those in public schools. These results suggest that a greater effort is needed to train teachers to deal with inclusion. In addition, public education centers should be provided with adequate resources to facilitate the possibilities of carrying out truly inclusive educational practices.

\section{Keywords}

Attitudes - Inclusive education - Teaching experience - Teacher training.

\section{Introducción}

La educación inclusiva, entendida como el derecho de todos los estudiantes a su presencia, participación y aprendizaje en los centros educativos (AINSCOW; BOOTH; DYSON, 2006), independientemente de cuáles sean sus capacidades, su sexo, lengua, origen étnico, estatus socioeconómico o cualquier otra condición, es uno de los elementos que se sitúan en el centro de la agenda de las políticas reformistas de numerosos sistemas educativos (OCDE, 2015; UNESCO, 2017). Estas reformas legales suponen propuestas políticas a nivel macro, que posteriormente deben materializarse en el día a día de las aulas, y transformarse en prácticas verdaderamente inclusivas (FINKELSTEIN; SHARMA; FURLONGER, 2019).

Sin embargo, en el tránsito entre lo que proponen los textos y reformas legales, a lo que verdaderamente se materializa en las aulas, influyen infinidad de factores que pueden favorecer o dificultar los procesos de inclusión (SLEE, 2018). Entre estos factores encontramos algunos como la formación del profesorado (HOPKINS; ROUND, 2018), o los recursos de que disponen para llevar a cabo las políticas inclusivas (MEIJER; WATKINS, 2019). Pero entre todos estos factores, uno de los conceptos que mayor atención ha recibido por parte de la investigación de este campo es el de las actitudes 
del profesorado hacia la educación inclusiva (ARMSTRONG, 2014; ROBERTS; SIMPSON, 2016; VAN MIEGHEM et al., 2018).

Las actitudes están constituidas por componentes cognitivos, que se refieren a las ideas previas o conocimientos sobre un aspecto determinado, componentes afectivos o sentimientos y finalmente componentes conductuales o predisposiciones para actuar o comportarse de determinada manera frente al aspecto en cuestión (EAGLY; CHAIKEN, 1993). En consecuencia, las actitudes implican posturas hacia conceptos, objetos e individuos, las cuales pueden ser favorables o desfavorables, y en el caso de la educación inclusiva, tienen un impacto en la forma en que los docentes apoyan a los estudiantes con necesidades educativas especiales (NEE) en los procesos escolares (LOREMAN, 2014).

A continuación, se abordan algunos de estos factores que juegan un papel relevante en la configuración de actitudes del profesorado hacia la inclusión, y que han sido estudiados por investigaciones previas.

\section{Variables demográficas, profesionales y escolares que afectan las actitudes hacia la inclusión en NEE}

Los factores demográficos y profesionales del profesorado, las condiciones de las aulas y el tipo de centro han sido analizados por su fuerte implicación con las actitudes presentadas por los maestros hacia la educación inclusiva. Los factores demográficos más estudiados han sido el sexo y la edad. En cuanto al sexo, los trabajos de revisión han encontrado resultados opuestos. Diversos estudios reportan que las maestras suelen tener actitudes más favorables (AVRAMIDIS; BAYLISS; BURDEN 2000; SALOVIITA; SCHAFFUS, 2016), otros no encuentran relación con el sexo (HAYDAR; MEDIA, 2010; CLAVIJO et al., 2016), y los metaanálisis han llegado a concluir que la evidencia es más bien inconsistente (AVRAMIDIS; NORWICH 2002; BOER; PIJL; MINNAERT, 2011).

Un gran número de estudios y revisiones exhaustivas que han analizado la relación edad-actitudes, han encontrado que los profesores más jóvenes tienen actitudes más positivas (AVRAMIDIS; NORWICH 2002; BOER; PIJL; MINNAERT, 2011; CLAVIJO et al., 2016; SALOVIITA; SCHAFFUS, 2016, TSAKIRIDOU; POLYZOPOULOU, 2014). Al parecer, los maestros con mayores años de trabajo presentan una tendencia hacia perpetuar prácticas que podían calificarse como escasamente inclusivas (BOER PIJL; MINNAERT, 2011).

La experiencia previa con estudiantes con NEE, así como la formación profesional han demostrado ser factores profesionales estrechamente relacionados con actitudes positivas del profesorado hacia la inclusión (AVRAMIDIS; NORWICH, 2002; BLACKMAN; CONRAD; BROWN, 2012; BATSIOU et al., 2008; BOER PIJL; MINNAERT, 2011; YEO et al., 2014). $\mathrm{Al}$ parecer, cuando las experiencias educativas previas con estudiantes con NEE obtienen resultados positivos, los profesores adquieren mayor confianza y menos resistencia hacia este tipo de prácticas inclusivas (AHMMED; SHARMA; DEPPELER 2012; KURNIAWATI et al., 2017; TSAKIRIDOU; POLYZOPOULOU, 2014). En los estudios presentados se puede destacar que la formación profesional y la experiencia que se genera como resultado de la implementación seria, sistematizada y controlada de la educación inclusiva, son los factores que mayor impacto tienen sobre las actitudes hacia la inclusión (KYONG-AH; SOO-YOUNG; 
HYUN-J00, 2017). No obstante, si bien los maestros pueden tener una actitud más positiva y optimista por la inclusión, también les preocupa la disponibilidad de recursos y apoyos para garantizar el éxito de este proceso (OSWALD; SWART, 2011).

Precisamente los recursos son parte de las condiciones de las aulas que afectan las actitudes. Los recursos son los apoyos adicionales de maestros especializados, los materiales didácticos y accesibilidades con las que cuenta la escuela para desarrollar sus procesos de inclusión. La disposición de este tipo de ayudas y materiales puede favorecer las actitudes de los maestros (MONSEN; EWING; KWOKA, 2014). Por otro lado, la falta de estos apoyos puede ser una razón poderosa para que los maestros muestren valoraciones negativas hacia la educación inclusiva (SALOVIITA; SCHAFFUS, 2016). Respecto a este tema, se ha planteado que la demanda hacia estos recursos suele ser poco realista y exagerada, especialmente si los profesores no tienen suficiente formación y experiencia en procesos inclusivos (DIAS; CADIME, 2015; EWING; MONSEN; KIELBLOCK, 2018).

La baja cantidad de niños con NEE por clase tiene efectos positivos para la inclusión (LÜBKE; PINQUART; SCHWINGER, 2019) pues los profesores manifiestan que es más complicado gestionar sus recursos y resolver sus necesidades diversas cuando aumenta la cantidad de niños con necesidades complejas (GOODMAN; BURTON, 2010). Además, la alta tasa de alumnado por aula sumada a instalaciones limitadas y a exceso de trabajo administrativo genera preocupaciones y resistencia hacia la educación inclusiva (KURNIAWATI et al., 2017).

Finalmente, se ha podido apreciar que la actitud de los profesores tiene que ver con el tipo de centro. Algunos trabajos han encontrado que en los centros de carácter privado se exteriorizan actitudes más positivas hacia la inclusión que en los centros públicos (CLAVIJO et al., 2016). En otros casos los centros concertados han demostrado tener mayor formación y recursos hacia la inclusión que los centros públicos y mayores apoyos personales para la implementación del modelo inclusivo que los centros privados (GARZÓN; CALVO; ORGAZ, 2016).

Dado que la literatura científica ha evidenciado de manera consistente que las actitudes del profesorado son un factor relevante para posibilitar que las políticas inclusivas puedan derivar de manera efectiva en prácticas verdaderamente inclusivas, en el presente estudio planteamos como objetivo analizar las actitudes hacia la inclusión educativa en una muestra de docentes de la ciudad de Cuenca (Ecuador).

Consideramos que se trata de un objetivo relevante y pertinente en este momento, dado que los resultados del estudio pueden contribuir a entender mejor las condiciones reales que se dan en las escuelas de Ecuador en el actual escenario, en que la Ley Orgánica de Educación Intercultural (LOEI) publicada en el año 2011 plantea como una de sus prioridades la necesidad de dotar a la educación de un carácter verdaderamente inclusivo.

\section{Método}

El estudio es de tipo transversal, descriptivo-comparativo realizado con una muestra aleatoria no representativa de docentes de escuelas públicas y privadas de la ciudad de Cuenca (Ecuador). 


\section{Participantes}

Participaron 66 docentes de 15 centros educativos seleccionados aleatoriamente. El 48.50\% de los asistentes pertenecían a instituciones públicas y el 51.50\% a instituciones privadas. Las edades de los participantes oscilaron desde 21 hasta 64 años ( $\bar{x}=40.29$; $\mathrm{O}=12.06^{4}$ ). Las características de los participantes en función del tipo de centro se exponen a continuación:

Tabla 1- Características de los profesores en función del tipo de institución

\begin{tabular}{|c|c|c|}
\hline & \multicolumn{2}{|c|}{ Tipo de Institución } \\
\hline & Pública & Privada \\
\hline \multicolumn{3}{|l|}{ Nivel de Formación \% } \\
\hline Bachiller & $3.12 \%$ & $5.80 \%$ \\
\hline \multicolumn{3}{|l|}{ Técnico } \\
\hline Ciencias de la educación & $3.12 \%$ & \\
\hline Mecánica automotriz & & $2.94 \%$ \\
\hline Tecnólogo (en Ciencias de la Educación) & $18.75 \%$ & $17.64 \%$ \\
\hline Profesor & $37.50 \%$ & $12.70 \%$ \\
\hline \multicolumn{3}{|l|}{ Licenciado en } \\
\hline Ciencias de la educación & $34,30 \%$ & $32,60 \%$ \\
\hline Psicología & & $7,54 \%$ \\
\hline Diplomado en ciencias de la educación & & $14.90 \%$ \\
\hline Máster en ciencias de la educación & $3.21 \%$ & $5.88 \%$ \\
\hline Total & $100 \%$ & $100 \%$ \\
\hline $\operatorname{Edad}(\sigma)$ & 43.44 & 37.76 \\
\hline Sexo\% & (11.29) & (12.09) \\
\hline Hombres & $34.37 \%$ & $20.6 \%$ \\
\hline Mujeres & $65.62 \%$ & $79.4 \%$ \\
\hline Total & $100 \%$ & $100 \%$ \\
\hline
\end{tabular}

$\overline{\mathrm{X}}=$ media; $\mathrm{O}=$ desviación típica

Fuente: Datos de investigación.

Las titulaciones de los participantes son en su mayoría en Ciencias de la educación. Esta titulación es un término genérico que sirve para referirse a las carreras afines al área de educación como son normalistas, pedagogos, profesores y licenciados con menciones en educación inicial, básica o media y gestión educativa, entre otros. 


\section{Instrumentos}

Utilizamos una adaptación de la escala ORI: Opinions Relative to Integration of Students with Disabilities Scale (ANTONAK; LARRIVEE, 1995), un cuestionario tipo Likert de veintitrés ítems agrupados en cinco factores que en conjunto buscan medir en los maestros las actitudes hacia la inclusión. Los cinco factores son: 1. Beneficios de la inclusión (ejemplo: la conducta de los estudiantes con NEE es un mal ejemplo para sus compañeros/as sin NEE); 2. Atención generalista vs. atención especialista (ejemplo: el reto de estar en un aula generalista fomenta el progreso académico de los estudiantes con NEE); 3. Metodología y manejo de la conducta en el aula (ejemplo: los estudiantes con NEE no están socialmente aislados en las aulas generalistas); 4. Esfuerzo $y$ dedicación del profesorado (ejemplo: los estudiantes con NEE no monopolizan el tiempo que el profesor generalista dedica a sus estudiantes); y 5. Formación y competencia del profesorado (ejemplo: los profesores generalistas tienen suficiente formación para enseñar a estudiantes con NEE). Estos factores explican en conjunto el 55.12\% de la varianza total del cuestionario.

La prueba tiene características psicométricas apropiadas con un coeficiente de Spearman-Brown de correlación entre dos mitades de .87 y un Alfa de Cronbach de .82 . El cuestionario ofrece una puntuación global hacia la inclusión educativa, así como una puntuación para cada uno de los factores que lo componen. Cada item presenta cinco opciones de respuesta: 1 = muy de acuerdo, $2=$ de acuerdo, $3=$ indiferente, $4=$ en desacuerdo y 5 = muy en desacuerdo. Trece de los items son de sentido directo, es decir, la respuesta muy de acuerdo implica una actitud positiva hacia la inclusión, mientras que los doce ítems restantes son de sentido inverso; en estos casos la respuesta muy en desacuerdo indica una actitud más favorable a la inclusión.

Antes de su aplicación, la escala fue revisada por dos maestros de escuela y dos profesores universitarios para adecuar las expresiones y la terminología al contexto cultural de Ecuador.

\section{Procedimiento}

Esta investigación pertenece a un estudio realizado con 2200 niños de CuencaEcuador, sus maestros, padres de familia y directores, que valoró indicadores de inclusión entre los que se encuentran el objetivo de este estudio.

Para apoyar la recolección de la información se capacitó a quince profesionales en Ciencias de la educación quienes tuvieron formación en educación inclusiva y atención a las diversas NEE. Se revisó con este equipo el procedimiento para explicar el desarrollo del cuestionario de manera que el proceso de aplicación de la encuesta evitara dirigir al encuestado hacia la adopción de un punto de vista. Se solicitaron los respectivos consentimientos a los profesores que participarían en esta investigación. La encuesta fue cumplimentada por el maestro, en presencia del equipo de apoyo para asistirle en caso de que surgieran dudas sobre las preguntas. 


\section{Análisis de datos}

El software utilizado para la realización de los estadísticos fue el paquete SPSS 24.00. Se realizaron análisis de varianza univariado ANOVA.

\section{Resultados}

Para facilitar la comparación entre factores, en todos los análisis las puntuaciones se han normalizado en una escala de 1 a 5 , dividiendo la puntuación de cada factor entre el número de ítems que lo componen. Las puntuaciones más bajas indican una mejor actitud hacia la inclusión y las puntuaciones más altas indican una peor actitud hacia la inclusión, quedando los valores próximos a 3 como actitudes neutras o indiferentes.

Las medias y desviaciones típicas resultantes de cada factor se presentan en la en la siguiente tabla:

Tabla 2- Medias de los factores de la escala de actitudes

\begin{tabular}{c|c|c}
\hline Factor & $\overline{\mathrm{X}}$ & $\sigma$ \\
\hline Beneficios de la inclusión & 2.25 & $(.18)$ \\
\hline Atención generalista vs. atención especialista & 1.89 & $(.54)$ \\
\hline Metodología y manejo de la conducta en el aula & 1.54 & $(.36)$ \\
\hline Esfuerzo y dedicación del profesorado hacia los estudiantes con NEE & 2.27 & $(.46)$ \\
\hline Formación y competencia del profesorado & 2.36 & $(.44)$ \\
\hline
\end{tabular}

$\overline{\mathrm{X}}=$ media; $\sigma=$ desviación típica

Fuente: Datos de investigación.

\section{Las actitudes de los profesores en función de las variables demográficas del profesorado}

La comparación entre las puntuaciones según el sexo (48 mujeres y 18 hombres) de los participantes a través del análisis de varianza no mostró diferencias estadísticamente significativas en ninguno de los cinco factores, Beneficios de la inclusión $\left(F_{(1,65)}=.39, p=.534\right)$; Atención generalista vs. especialista $\left(F_{(1,65)}=2.64, p=.108\right)$; Metodología y manejo de conducta $\left(F_{(1,65)}=1.23, p=.270\right)$; Esfuerzo hacia estudiantes con NEE $\left(F_{(1,65)}=.86, p=.355\right)$; Formación y competencias $\left(F_{(1,65)}=1.61, p=.208\right)$.

La comparación de los tres rangos de edad de los participantes $(21-35 ; 36-50 ; \geq 51)$ mostró valores significativos con Atención generalista vs. especialista $\left(F_{(1,65)}=.373\right.$, $p=.004)$. No se encontraron diferencias estadísticamente significativas para las otros factores (Beneficios de la inclusión, $F_{(1,65)}=.125, p=.355$; Metodología y manejo de conducta, $F_{(1,65)}=-.001, p=.997$; Esfuerzo hacia estudiantes con NEE, $F_{(1,65)}=.110, p=.407$; Formación y competencias, $\left.F_{(1,65)}=.187, p=.150\right)$. 


\section{Las actitudes de los profesores en función de las variables profesionales del profesorado}

La variable experiencia en docencia con estudiantes que presentan NEE mostró diferencias significativas únicamente en el Factor Atención generalista vs. especialista, en el sentido de que el grupo sin experiencia con NEE mostró una peor actitud hacia esta variable, como se presenta a continuación:

Tabla 3- Diferencia en las actitudes hacia la inclusión entre profesorado con (Si) y sin (No) experiencia en docencia a estudiantes con NEE

\begin{tabular}{|c|c|c|c|c|c|}
\hline Factor & $\begin{array}{c}\text { Sí } \\
\mathrm{n}=36 \\
\overline{\mathbf{x}}\end{array}$ & $\begin{array}{c}\text { No } \\
\mathrm{n}=30 \\
\overline{\mathbf{x}}\end{array}$ & $F_{(1,65)}$ & $P$ & $\eta^{2} p$ \\
\hline Beneficios de la inclusión & 2.23 & 2.31 & 2.97 & .089 & .044 \\
\hline Generalista vs. especialista & $\begin{array}{l}1.85 \\
(.53)\end{array}$ & $\begin{array}{l}2.14 \\
(.54)\end{array}$ & 4.64 & $.035^{*}$ & .068 \\
\hline Metodología y manejo conducta & $\begin{array}{l}1.54 \\
(.42)\end{array}$ & $\begin{array}{l}1.54 \\
(.31) \\
\end{array}$ & 0.00 & 1.00 & .000 \\
\hline Esfuerzo hacia estudiantes NEE & $\begin{array}{l}2.18 \\
(.51)\end{array}$ & $\begin{array}{l}2.37 \\
(.38)\end{array}$ & 2.85 & .096 & .043 \\
\hline Formación y competencia & $\begin{array}{l}2.37 \\
(.49)\end{array}$ & $\begin{array}{l}2.34 \\
(.49)\end{array}$ & .093 & .761 & .001 \\
\hline
\end{tabular}

${ }^{\star *} p<.01,{ }^{*} p<.05$

$\overline{\mathrm{X}}=$ media

Fuente: Datos de investigación.

En cuanto a los años de experiencia como maestros se generaron tres grupos: 1 a 5 años de experiencia, 6 a 11 años de experiencia y más de 11 años de experiencia. Realizamos ANOVAs y no obtuvimos diferencias estadísticamente significativas (Beneficios de la inclusión, $F_{(1,65)}=1.62, p=.204$; Atención generalista vs. especialista, $F_{(1,65)}=2.04, p=.138 ;$ Metodología y manejo de conducta, $F_{(1,65)}=.041, p=.960 ;$ Esfuerzo hacia estudiantes con NEE, $F_{(1,65)}=1.20, p=.308$; Formación y competencias, $F_{(1,65)}=.222$, $p=.802)$.

Analizamos el nivel de formación de los participantes. Se generaron siete grupos: Bachilleres, que se refiere a quienes han finalizado sus estudios de colegio; Técnicos, quienes tienen una formación profesionalizante de dos años; Tecnólogos, con una formación profesionalizante de tres años; Profesores, quienes se han formado en institutos pedagógicos, es decir, en planteles post-bachillerato con tres años de estudio teórico y práctico; Licenciados, quienes han estudiado una carrera universitaria completa; Especialistas, que se refiere a una capacitación profesional avanzada a nivel de postgrado; y Magister, que se refiere a una formación completa en este mismo nivel.

Tras realizar los correspondientes ANOVAs, no se encontraron diferencias estadísticas en ninguno de los factores, para ninguno de los grupos (Beneficios de la inclusión, $F_{(1,65)}=1.18$, 
$p=.325$; At. generalista vs. especialista, $F_{(1,65)}=0.767, p=.599 ;$ Metodología y manejo de conducta, $F_{(1,65)}=1.03, p=.409 ;$ Esfuerzo hacia estudiantes con $N E E, F_{(1,65)}=.478, p=.822$; Formación y competencias, $\left.F_{(1,65)}=2.19, p=.056\right)$.

\section{Las actitudes de los profesores en función del tipo de centro}

Se realizaron las comparaciones en función del tipo de escuela (pública y privada) a través del análisis de varianza, resultando estadísticamente significativo el análisis del factor 5 Formación y competencia del profesorado, en el sentido de que los profesores de escuelas públicas mostraron una peor actitud hacia este factor.

Los resultados del ANOVA para tipo de escuela se presentan en la siguiente tabla:

Tabla 4- Diferencias en las actitudes hacia la inclusión entre escuelas públicas y privadas

\begin{tabular}{|c|c|c|c|c|c|}
\hline & \multicolumn{2}{|c|}{ Tipo de escuela } & \multirow{3}{*}{$F_{(1,65)}$} & \multirow{3}{*}{$P$} & \multirow{3}{*}{$\eta^{2} p$} \\
\hline & $\begin{array}{c}\text { Pública } \\
\text { n=32 } \\
\overline{\mathbf{X}}\end{array}$ & $\begin{array}{c}\text { Privada } \\
\mathrm{n}=34 \\
\overline{\mathbf{X}}\end{array}$ & & & \\
\hline & $\sigma$ & $\sigma$ & & & \\
\hline Beneficios de la inclusión & $\begin{array}{l}2.30 \\
(.14)\end{array}$ & $\begin{array}{l}2.23 \\
(.22)\end{array}$ & 2.51 & .118 & .038 \\
\hline Generalista vs. especialista & $\begin{array}{l}2.09 \\
(.50) \\
\end{array}$ & $\begin{array}{l}1.88 \\
(.58) \\
\end{array}$ & 2.44 & .122 & .037 \\
\hline Metodología, manejo conducta & $\begin{array}{l}1.60 \\
(.34)\end{array}$ & $\begin{array}{l}1.48 \\
(.39)\end{array}$ & 1.61 & .209 & .025 \\
\hline Esfuerzo y dedicación hacia estudiantes con NEE. & $\begin{array}{l}2.31 \\
(.40)\end{array}$ & $\begin{array}{l}2.23 \\
(.52)\end{array}$ & .446 & .507 & .007 \\
\hline Formación y competencia & $\begin{array}{l}2.48 \\
(.43)\end{array}$ & $\begin{array}{l}2.24 \\
(.46)\end{array}$ & 4.86 & $.031^{*}$ & .071 \\
\hline
\end{tabular}

${ }^{\star *} p<.01,{ }^{*} p<.05$

$\overline{\mathrm{X}}=$ media; $\sigma=$ desviación típica

Fuente: Datos de investigación.

\section{Discusión}

Los resultados de la valoración de las actitudes de los maestros de educación primaria hacia la educación inclusiva mostraron una tendencia tan solo tímidamente favorable hacia las posiciones más inclusivas. Aunque este resultado supone un ligero avance respecto al único trabajo que hasta la fecha ha analizado las actitudes del profesorado hacia la inclusión en Ecuador (CLAVIJO et al., 2016), todavía se encuentra muy alejado de la situación a la que aspiran las políticas promovidas por la LOEI (ECUADOR, 2011) en Ecuador.

La valoración de este resultado no puede ser en absoluto positiva, ya que muestra todavía actitudes muy alejadas de lo que sería una apuesta decidida por la inclusión y 
no sugiere ni mucho menos un convencimiento firme de los beneficios de la educación inclusiva, unas actitudes con las que difícilmente podemos esperar prácticas educativas adecuadas desde el punto de vista de la inclusión (SHARMA; JACOBS, 2016).

Con el objetivo de analizar los factores que pueden influir en la configuración de actitudes hacia la inclusión, en la presente investigación se han analizado tres tipos de variables que pueden ser relevantes en esta formación de actitudes: demográficas y profesionales del profesorado y el tipo de centro (público o privado) en que el profesorado realiza su labor educativa.

En lo que respecta a las variables demográficas, las investigaciones previas habian encontrado resultados inconsistentes, ya que en algunos estudios sí se había hallado una mejor actitud hacia la inclusión de las profesoras en comparación con los profesores, pero en otros casos no había sido así (ver las revisiones de Avramidis y Norwich (2002) y Boer, Pijl y Minnaert (2011)). Los resultados del presente estudio no hallaron diferencias en las actitudes del profesorado en función del sexo, lo que corrobora que no está claro que estas actitudes dependan del sexo de los docentes.

Sin embargo, sí hallamos una relación estadísticamente significativa entre la edad de los docentes y sus actitudes hacia la inclusión. Concretamente, la edad de los participantes se relacionaba positivamente con el factor Atención generalista vs. especialista, lo que sugiere que los profesores de mayor edad realizan mayores distinciones entre profesorado especialista de educación especial y generalista y consideran que la atención educativa a los estudiantes con NEE es responsabilidad fundamentalmente de los especialistas. Esta diferenciación entre profesorado especialista y generalista es probablemente uno de los puntos más controvertidos en el campo de la inclusión, ya que, si bien el profesorado especialista es imprescindible en los centros educativos para atender a los estudiantes con mayores NEE, en algunas ocasiones su actuación va en contra de los principios de la inclusión, ya que los estudiantes con NEE se convierten en su responsabilidad, lo que automáticamente exime de esta responsabilidad al profesorado generalista, y genera situaciones poco deseables de segregación dentro de los propios centros educativos (MONTGOMERY; MIRENDA, 2014). Este resultado es similar al encontrado por algunos estudios previos que también habían encontrado mejores actitudes hacia la inclusión en los docentes más jóvenes (CLAVIJO et al., 2016), y es parcialmente coincidente con el estudio de Rakap y Kaczmarek (2010), que encontró diferencias significativas en dos grupos extremos, es decir, en los más jóvenes y en los más veteranos.

En cuanto a las variables profesionales de los docentes, en el presente estudio no se encontraron resultados estadísticamente significativos en las variables de experiencia docente y nivel de formación, pero sí en la variable de experiencia docente con estudiantes con NEE, nuevamente en la escala de atención generalista vs. especialista, que, tal y como ocurría con la variable edad, se relaciona negativamente con las actitudes hacia la inclusión. En otras palabras, parece que los resultados del estudio sugieren que los docentes sin experiencia con estudiantes con NEE incidian más en la distinción entre las funciones del profesorado especialista, que debe hacerse responsable de la educación de los estudiantes con NEE, y el profesorado generalista, que delega en el anterior su responsabilidad con los 
niños con NEE, un resultado diametralmente opuesto a los preceptos de la inclusión, ya que es el primer paso para generar prácticas segregadoras en absoluto inclusivas.

La ausencia de relación entre los años de experiencia docente y las actitudes hacia la inclusión es un resultado que también se ha encontrado en otros estudios previos en los que los años de experiencia docente tampoco se han revelado como un factor que ejerza una influencia significativa en las actitudes hacia la inclusión (AVRAMIDIS; BAYLISS; BURDEN, 2000). Sin embargo, otros estudios sí han encontrado esta influencia. Así, los profesores con menos años de experiencia apoyaban más el modelo inclusivo (AVRAMIDIS; NORWICH, 2002; BOER; PIJL; MINNAERT, 2011), mientras que en otros casos estas condiciones se apreciaban en los profesores con más de 10 años de experiencia (CLAVIJO et al., 2016; RAKAP; KACZMAREK, 2010).

La ausencia de influencia de los años de experiencia en las actitudes demostradas hacia la inclusión en el presente estudio podría explicarse porque el modelo de educación inclusiva se introduce formalmente en Ecuador a raíz de la LOEI (ECUADOR, 2011) y, al ser relativamente reciente en este sistema educativo, no se puede apreciar una diferencia entre los profesores recién formados y que poseen menores años de experiencia en el magisterio, con los profesores más experimentados y con una formación más antigua.

En cuanto a la formación del profesorado, llama la atención que existan todavía algunos casos (concretamente un 4.46\%) de bachilleres en las instituciones públicas y privadas, es decir, graduados del colegio que no tienen formación universitaria o que aún no han obtenido su titulación universitaria, una condición que en la literatura científica se denomina profesores empíricos, es decir, profesores que no han estudiado carreras relacionadas con la docencia o no se han graduado como maestros (VAILLANT, 2005).

La formación del profesorado debería ser una de las prioridades de un sistema educativo como el ecuatoriano que aspira a modernizarse y a alcanzar una situación equiparable a la de otros países occidentales. Sin embargo, los resultados de las investigaciones que han analizado esta variable muestran que la formación del profesorado, lejos de mejorar, parece haber empeorado en las últimas décadas. Si hacia finales del siglo pasado el Banco Mundial reportaba que cerca del 10\% del personal que desempeñaba las funciones de magisterio en Ecuador eran profesores empíricos (ÁLVAREZ; MAJMUDAR, 2001), en 2016 un informe similar aumentaba esta cifra al 18.4\% (BANCO MUNDIAL, 2016), una situación muy alejada de la deseable que dificulta la mejora del sistema educativo en general, y que es particularmente perniciosa para los aspectos más sensibles de la educación, como el de la educación inclusiva.

De hecho, varias investigaciones previas han hallado una relación significativa entre la formación recibida por el profesorado sobre educación inclusiva y las actitudes hacia este modelo educativo. En concreto, el entrenamiento y formación profesional se han considerado como el éxito en la inclusión, pues disminuye la resistencia hacia las prácticas inclusivas (AVRAMIDIS; NORWICH, 2002; BOER; PIJL; MINNAERT, 2011). En el caso de Ecuador, esta formación específicamente relacionada con la educación inclusiva es escasa en los programas de formación inicial del profesorado, por lo que consideramos que puede estar afectando a los resultados del presente estudio. 
Finalmente, la última variable estudiada como elemento influyente en las actitudes hacia la inclusión fue el tipo de centro: los docentes de centros privados mostraron mejores actitudes hacia la inclusión en el quinto factor del instrumento, que hace referencia precisamente a la formación y competencia del profesorado. Es decir, los docentes de las escuelas privadas manifestaron que su formación y competencia profesional les permite atender con las suficientes garantías a niños con NEE en mayor medida que los docentes de las escuelas públicas. Estos resultados son similares a los que se encontraron en el estudio de Clavijo y otros autores (2016), también en el contexto ecuatoriano, quienes encontraron diferencias estadísticamente significativas a favor de las instituciones privadas. Ante estos resultados, consideramos pertinente preguntarnos si los maestros de las instituciones privadas están mejor capacitados para la inclusión, o bien si es la presencia de otros factores contextuales la que favorece y potencia la formación que estos tienen.

Habitualmente, los centros educativos privados en Ecuador escolarizan estudiantes de un nivel socioeconómico superior a los de los centros públicos (PEREYRA, 2008), probablemente debido a la posibilidad que tienen estos centros de seleccionar a su alumnado a través de diferentes mecanismos (ROSSETTI, 2014). Además, los centros privados también disponen de mayores recursos personales y materiales, lo que favorece la creación de mejores condiciones respecto a la organización de la enseñanza y el clima escolar (DUARTE; BOSS; MORENO, 2011) y aumenta las posibilidades de desarrollar prácticas educativas inclusivas (LÜBKE; PINQUART; SCHWINGER, 2019; SALOVIITA; SCHAFFUS, 2016).

Precisamente Granada, Pomés y Sanhueza (2013) plantean que varios factores afectan a la disposición y actitud de los docentes para que puedan hacer frente a las demandas que exige el modelo inclusivo. Entre ellas se encuentran la colaboración de la familia, el apoyo para generar estrategias diversificadas de enseñanza, el tiempo del que disponen, o los recursos para favorecer la inclusión (material didáctico, adaptaciones a los edificios, apoyo de especialistas). Por lo tanto, parece bastante probable que, en cierta medida, sean estos factores contextuales (y no necesariamente la formación del profesorado) los que afecten al mayor sentimiento de competencia del profesorado en las escuelas privadas.

Este estudio presenta algunas limitaciones. En primer lugar, el tamaño de la muestra empleada ha sido reducido, lo que puede haber influido en la ausencia de significación estadística en los diferentes análisis realizados, por lo que la interpretación de los datos debe realizarse con cautela. En segundo lugar, no se ha podido tener en cuenta algunas variables contextuales que podrían estar influyendo en las actitudes del profesorado hacia la inclusión, tales como la disposición de recursos y el apoyo recibido de la comunidad educativa. Consideramos que sería interesante valorarlas en trabajos de este tipo para medir la participación que estos factores tienen en las actitudes de los maestros. Finalmente, es posible que los resultados del presente estudio se hayan visto influidos por el sesgo de deseabilidad social, un sesgo cuya presencia se ha constatado en estudios previos relacionados con esta temática (LÜKE; GROSCHE, 2018), y cuya aparición además puede verse favorecida por los intensos procesos de evaluación, seguimiento y rendición de cuentas que se están produciendo a partir de la implantación de la LOEI, de manera que 
estas evaluaciones pueden producir que los docentes perciban que investigaciones como la del presente estudio supongan una nueva evaluación de su trabajo docente.

Finalmente, consideramos que tras la realización de este trabajo se abren algunas futuras vías de investigación relevantes que sería interesante explorar. Entre estos factores, se encuentra el análisis de los procesos de formación inicial y continua del profesorado en Ecuador (un factor que puede estar influyendo de manera relevante en la configuración de actitudes). Además, consideramos que sería positivo llevar a cabo estudios longitudinales para monitorizar (mediante metodologías cuantitativas), cómo evolucionan las actitudes del profesorado hacia la inclusión a lo largo del tiempo y para analizar (mediante metodologías cualitativas), qué factores determinan los posibles cambios actitudinales del profesorado.

\section{Referencias}

AHMMED, Masud; SHARMA, Umesh; DEPPELER, Joanne. Variables affecting teachers' attitudes towards inclusive education in Bangladesh. Journal of Research in Special Educational Needs, Amington, v. 12, n. 3 , p. 132-140, 2012. Doi: 10.1111/j.1471-3802.2011.01226.x

AINSCOW, Mel; BOOTH, Tony; DYSON, Alan. Improving schools, developing inclusion. London: Routledge, 2006.

ÁLVAREZ, Benjamin; MAJMUDAR, Julieta. ¿Quién está preparando a nuestros hijos para el siglo del conocimiento? Washington, DC: The World Bank, 2001. Human Development Department. (LCSHAD Paper Series; 67S).

ANTONACK, Richard; LARRIVEE, Bárbara. Psychometric analysis and revision of the opinions relative to mainstreaming scale. Exceptional Children, Arlington, v. 62, n. 2, p. 139-149, 1995. Doi: $10.1177 / 001440299506200204$

ARMSTRONG, David. Educator perceptions of children who present with social, emotional and behavioural difficulties: a literature review with implications for recent educational policy in England and internationally. International Journal of Inclusive Education, London, v. 8., n. 7, p. 731-745, 2014. Doi: $10.1080 / 13603116.2013 .823245$

AVRAMIDIS, Elias; BAYLISS, Phil; BURDEN, Robert. Student teachers' attitudes towards the inclusion of children with special educational needs in the ordinary school. Teaching and Teacher Education, London, v. 16, n. 3, p. 277-293, 2000. Doi: 10.1016/S0742-051X(99)00062-1

AVRAMIDIS, Elias; NORWICH, Brahm. Teachers' attitudes towards integration/inclusion: a review of the literature. European Journal of Special Needs Education, London, v. 17, n. 2, p. 129-147, 2002. Doi: $10.1080 / 08856250210129056$

BANCO MUNDIAL. Datos Ecuador. Washington, DC: [s. n.], 2016. Disponible en: <http://datos. bancomundial. org/pais/ecuador?view=chart>. Acceso en: 28 mar.2018. 
BATSIOU, Sofia et al. Attitudes and intention of Greek and Cypriot primary education teachers towards teaching pupils with special educational needs in mainstream schools. International Journal of Inclusive Education, London, v. 12, n. 2, p. 201-219, 2008. Doi: 10.1080/13603110600855739

BLACKMAN, Stacey; CONRAD, Dennis; BROWN, Launcelot. The attitude of Barbadian and Trinidadian teachers to integration. International Journal of Special Education, London, v. 21, n. 3, p. 158-168, 2012. Doi: https://eric.ed.gov/?id=EJ1001068

BOER, Anke; PIJL, Sip Jan; MINNAERT, Alexander. Regular primary schoolteacher' attitudes towards inclusive education: a review of the literature. International Journal of Inclusive Education, London, v. 15, n. 3, p. 331-353, 2011. Doi: 10.1080/13603110903030089

CLAVIJO, Ruth et al. Actitudes docentes hacia la educación inclusiva en Cuenca. Maskana, Cuenca, v. 7, n. 1, p. 13-22, 2016. Doi: 10.18537/mskn.07.01.02

DIAS, Paulo; CADIME, Irene. Effects of personal and profesional factors on teachers' attitudes towards inclusion in preschool. European Journal of Special Needs Education, London, v. 31, n. 1, p. 111-123, 2015. Doi: 10.1080/08856257.2015.1108040

DUARTE, Jesus; BOS, María Soledad; MORENO, Martín. Los docentes, las escuelas y los aprendizajes escolares en América Latina: un estudio regional usando la base de datos del SERCE. Washington, DC: BID, 2011. (Nota técnica \#8).

EAGLY, Alice; CHAIKEN, Shelly. The nature of attitudes. In: EAGLY, Alice; CHAIKEN, Shelly (Ed.). The psychology of attitudes. Fort Worth: Harcourt, Brace \& Janovich, 1993. p.1-21.

ECUADOR. Presidencia de la República. Ley Orgánica de Educación Intercultural de 31 de marzo de 2011. Registro Oficial, Quito, v. 2, Seg. supl., n. 147, 2011. Disponible en: <http://planipolis.iiep.unesco.org/ upload/Ecuador/Ecuador_Ley_organica_educacion_intercultural.pdf>. Acceso en: 10 dic. 2019.

EWING, Donna; MONSEN, Jeremy; KIELBLOCK, Stephan. Teachers' attitudes towards inclusive education: a critical review of published questionnaires. Educational Psychology in Practice, London, v. 34, n. 2, p. 150165, 2018. Doi: 10.1080/02667363.2017.1417822

FINKELSTEIN, Simon; SHARMA, Umesh; FURLONGER, Brett. The inclusive practices of classroom teachers: a scoping review and thematic analysis. International Journal of Inclusive Education, London, p. 1-28, 2019. Doi: 10.1080/13603116.2019.1572232

GARZÓN, Paula; CALVO, María Isabel; ORGAZ, Begoña. Inclusión educativa: actitudes y estrategias del profesorado. Revista Española de Discapacidad, Madrid, v. 4, n. 2, p. 25-45, 2016. Disponible en: <https:// dialnet.unirioja.es/servlet/articulo?codigo=5765598>. Acceso en: 18 en. 2020.

GOODMAN, Ruth; BURTON, Diana. The inclusion of students with BESD in mainstream schools: teachers' experiences of and recommendations for creating a successful inclusive environment. Emotional and Behavioural Difficulties, London, v. 15, n. 3, p. 223-237, 2010. Doi:10.1080/13632752.2010.497662 
GRANADA, Maribel; POMÉS, Maria; SANHUEZA, Susan. Actitud de los profesores hacia la inclusión educativa. Papeles de Trabajo, Rosario, v. 25, p. 51-59, 2013. Disponible en: <http://hdl.handle. net/2133/3301>. Acceso en: 14 nov. 2019.

HAYDAR, Orietta; MEDIA, Fabián. Percepción y actitudes hacia la inclusión educativa de los docentes de Soledad, Atlántico (Colombia), 2008. Zona Próxima, Barranquilla, v. 12, p. 12-39, 2010. Disponible en: $<$ http://manglar.uninorte.edu.co/jspui/handle/10584/4526k>. Acceso en: 4 dic. 2020.

HOPKINS, Sarah; ROUND, Penny. Building stronger teacher education programmes to prepare inclusive teachers. In: FITZGERALD, Ange, PARR, Graham; WILLIAMS, Judy (Ed.). Re-imagining professional experience in initial teacher education. Singapore: Springer, 2018. p. 55-66.

KURNIAWATI, Farida et al. Evaluating the effect of a teacher training programme on the primary teachers' attitudes, knowledge and teaching strategies regarding special educational needs. Educational Psychology, London, v. 37, n. 3, p. 287-297, 2017. Doi: 10.1080/01443410.2016.1176125

KYONG-AH, Kwon; SO0-YOUNG, Hong; HYUN-J00, Jeon. Classroom readiness for successful inclusion: teacher factors and preschool children's experience with and attitudes toward peers with disabilities. Journal of Research in Childhood Education, London, v. 31, n. 3, p. 360-378, 2017. Doi: 10.1080/02568543.2017.1309480

LOREMAN, Tim. Measuring inclusive education outcomes in Alberta, Canada. International Journal of Inclusive Education, London, v. 18, n. 5, p. 459-483, 2014. Doi: 10.1080/13603116.2013.788223

LÜBKE, Laura; PINQUART, Martin; SCHWINGER, Malte. How to measure teachers' attitudes towards inclusion: evaluation and validation of the Differentiated Attitudes Towards Inclusion Scale (DATIS). European Journal of Special Needs Education, London, v. 34, n, 3, p. 297-311, 2019. Doi: 10.1080/08856257.2018.1479953

LÜKE, Timo; GROSCHE, Michael. What do I think about inclusive education? It depends on who is asking: experimental evidence for a social desirability bias in attitudes towards inclusion. International Journal of Inclusive Education, London, v. 22, n. 1, p. 38-53, 2018. Doi: 10.1080/13603116.2017.1348548

MEIJER, Cor; WATKINS, Amanda. Financing special needs and inclusive education-from Salamanca to the present. International Journal of Inclusive Education, London, v. 23, p. 1-17, 2019. Doi: 10.1080/13603116.2019.1623330

MONSEN, Jeremy; EWING, Donna; KWOKA, Maria. Teachers' attitudes towards inclusion, perceived adequacy of support and classroom learning environment. Learning Environments Research, Netherlands, v. 17, p. 1, p. 113-126, 2014. Doi: 10.1007/s10984-013-9144-8

MONTGOMERY, Angela; MIRENDA, Pat. Teachers' self-efficacy, sentiments, attitudes and concerns about the inclusion of students with developmental disabilities. Exceptionality Education International, New York, v. 24, n. 1, p-18-32, 2014. 
OCDE. Organización para la Cooperación y Desarrollo Económico. Education policy outlook 2015: making reforms happen. Paris: OCDE, 2015.

OSWALD, Marietjie; SWART, Estelle. Addressing South African pre-service teachers' sentiments, attitudes and concerns regarding inclusive education. International Journal of Disability, Development and Education, London, v. 58, n. 4, p. 389-403, 2011. Doi: 10.1080/1034912X.2011.626665

PEREYRA, Ana. La fragmentación de la oferta educativa en América Latina: la educación pública vs. la educación privada. Perfiles Educativos, Coyoacán, v. 30, n. 120, p. 132-146, 2008. Disponible en: <http:// www.scielo.org.mx/scielo.php?pid=S0185-26982008000200008\&script=sci_arttext>. Acceso en: 12 dic. 2019.

RAKAP, Salih; KACZMAREK, Louise. Teachers' attitudes towards inclusion in Turkey. European Journal of Special Needs Education, London, v. 25, n. 1, p. 59-75, 2010. Doi: 10.1080/08856250903450848

ROBERTS, Jacqueline; SIMPSON, Kate. A review of research into stakeholder perspectives on inclusion of students with Autism in Mainstream Schools. International Journal of Inclusive Education, London, v. 20, n. 10, p. 1084-1096, 2016. Doi: 10.1080/13603116.2016.1145267

ROSSETTI, Magdalena. La segregación escolar como un elemento clave en la reproducción de la desigualdad. Santiago de Chile: Unesco, 2014.

SALOVIITA, Timo; SCHAFFUS, Tina. Teacher attitudes towards inclusive education in Finland and Brandenburg, Germany and the issue of extra work. European Journal of Special Needs Education, London, v. 31, n. 4, p. 458-471, 2016. Doi: 10.1080/08856257.2016.1194569

SHARMA, Umesh; JACOBS, Kate. Predicting in-service teachers' intention to teach in inclusive classrooms in India and Australia. Teaching and Teacher Education, Amsterdan, v. 55, p. 13-23, 2016. Doi: 10.1016/j. tate.2015.12.004

SLEE, Roger. Inclusive education: from policy to school implementation. In: CLARK, Catherine; DYSON, Alan; MILLWARD, Alan (Ed.). Towards inclusive schools? London: Routledge. 2018. p. 30-41.

TSAKIRIDOU, Helen; POLYZOPOULOU, Konstantia. Greek teachers' attitudes toward the inclusion of students with special educational needs. American Journal of Educational Research, Newark, v. 2, n. 4, p. 208-218, 2014. Doi: 10.12691/education-2-4-6

UNESCO. A guide for ensuring inclusion and equity in education. Paris: Unesco, 2017.

VAILLANT, Denise. Reformas educativas y rol de docentes. Revista Prelac, Santiago de Chile, v. 1, p. 38-51, 2005.

VAN MIEGHEM, Aster et al. An analysis of research on inclusive education: a systematic search and meta review. International Journal of Inclusive Education, London, p. 1-15, 2018. Doi: 10.1080/13603116.2018.1482012 
YEO, Lay et al. Teachers' experience with inclusive education in Singapore. Asia Pacific Journal of Education, Singapur, v. 36, p. 69-83, 2014. Doi: 10.1080/02188791.2014.934781

Recibido en: 02.10.2019

Revisado en: 19.02 .2020

Aprobado en: 03.03.2020

Raúl Tárraga-Mínguez es doctor en Psicopedagogía, diplomado en Magisterio, licenciado en Psicopedagogía y en Antropología social y cultural y graduado en Pedagogía y Psicología. Profesor titular en la Universidad de Valencia (España).

Ximena Vélez-Calvo es doctora por la Universidad de Valencia, magíster en Educación parvularia, licenciada en Ciencias de la Educación (mención en lengua extranjera: inglés). Es profesora en la Universidad del Azuay (Ecuador).

Gemma Pastor-Cerezuela es doctora en Psicología y licenciada en Psicología con premio extraordinario de licenciatura y de doctorado. Profesora titular en la Universidad de Valencia (España).

María Inmaculada Fernández-Andrés es doctora en Psicopedagogía, con premio extraordinario de doctorado, licenciada en Psicopedagogía y diplomada en Magisterio. Profesora titular en la Universidad de Valencia (España). 